\title{
Kalman Filter to Improve Performance of PID Control Systems on DC Motors
}

\author{
Seta Yuliawan ${ }^{1}$, Oyas Wahyunggoro ${ }^{2}$, Nurman Setiawan ${ }^{3}$
}

\begin{abstract}
A proportional-integral-derivative (PID) controller is a type of control system that is most widely applied in industrial world. Various tuning models have been developed to obtain optimal performance in PID control. However, the methods are designed under ideal circumstances. This means that the control system which has been built will not work optimally when noise exists. Noise can come from electrical vibrations, inference of electronic components, or other noise sources. Thus, it is necessary to design PID control system that can work optimally without being disturbed by noise. In this research, Kalman filter was used to improve the performance of PID controllers. The application of Kalman filter was used to reduce the noise of the input signal so that it could generate output signal which is in accordance with the expected output. Simulation result showed that the PID performance with Kalman filter was more optimal than the ordinary one to minimize the existing noise. The resulting speed of DC motor with Kalman filter had a lower overshoot than PID control without Kalman filter. This method resulted lower integral of absolute error (IAE) than ordinary PID controls. The IAE value for the PID controller with the Kalman filter was 25.4, the PID controller with the observer was 31.0, while the IAE value in the ordinary controller was only 60.9 .
\end{abstract}

Keywords-Kalman Filter, PID, Noise, DC Motors.

\section{INTRODUCTION}

A proportional-integral-derivative (PID) controller is one type of controller that is widely applied in industrial control processes [1]. Although PID controllers have a simple structure as well as are easy to understand and apply to various control systems [2], PID controllers still has drawbacks. The PID control system's performance is primarily determined by tuning the PID parameters, namely $K p, K i$, and $K d$. Improper tuning will cause the performance of the controlled system to be suboptimal or even cause instability.

Recently, researchers have developed various methods of tuning the PID parameters. The development of various tuning methods aims to get optimal responses according to the desired design specifications. There are various tuning methods such as the Ziegler-Nichols method [3], ant colony [4], fuzzy [5], iterative feedback [6], and observer [7]. Even though these methods have worked well, they are designed under ideal circumstances; it means that the built control systems will not work optimally when there is noise. In the field, a lot of noise

${ }^{1,2}$ Department of Electrical and Information Engineering, Faculty of Engineering, Universitas Gadjah Mada, Jln. Grafika No. 2, Kampus UGM, Yogyakarta, 55281, INDONESIA (phone: 0274-552305; email: $\quad{ }^{1}$ seta.yuliawan@mail.ugm.ac.id, ${ }^{2}$ oyas@ugm.ac.id)

3 Department of Electrical Engineering, Faculty of Engineering, Yogyakarta State University, Karangmalang Campus, Yogyakarta, 55281, INDONESIA (phone: 0274-586168; email: ${ }^{3}$ nurman.setiawan@uny.ac.id) will disturb the system. Noise can come from the vibrations of electric motor, inference of electronic components, or other noise sources. Thus, it is necessary to design a PID control system that can work optimally without being disturbed by noise.

The factors that cause noise in the system are divided into two factors, namely external and internal factors. External factors are the sources of noise coming from other devices, as previously mentioned. Meanwhile, the internal factors are the sources of noise originating from Kalman filter which work like an estimator by considering covariance noise, both process noise and output noise. Kalman filter has a good performance in filtering noise. On the other hand, PID control will work well if there is no noise. The combination of PID control and Kalman filter is expected to overcome the decline in control system performance in the industrial world.

Plants or actuators that are widely used in industry are DC motors. DC motors can be modeled with a second order system. In the industrial world, DC motors must work with a fast response time without excessive overshoot. Fast response time will affect the efficiency of production time. Meanwhile, overshoot must be avoided so that there is no backlash on the gear and component damage can be minimized.

The control system performance that has been built must be tested and compared with other control systems. One of the parameters used to measure this performance is called integral of absolute error (IAE). IAE integrates absolute error over time [6].

This research aims to minimize the noise in the DC motor PID control system so that the control system has better performance. This research designs a PID control system for DC motors. Kalman filter is utilized to handle noise problems that often arise in the field. The combination of PID and Kalman filter is expected to make the DC motor have better performance without being disturbed by the noise that appears.

\section{Mathematical Model of DC Motor}

DC motors are the actuators that are mostly used in the industrial world. They convert electrical energy into rotational energy. When DC motors are combined with pulleys and belts, they will produce translational motion. Electronically, a DC motor consists of a field circuit and an armature circuit [8]. DC motor modeling based on [8] can be seen in Fig. 1. The torque $(\tau)$ generated by the DC motor is proportional to the current $(i)$ induced by the input voltage.

$$
\tau(t)=K m i(t)
$$

where $K_{m}$ is the armature constant which value depends on the motor's physical properties, such as the number of turns in the conducting coil, the magnetic field strength, and so on.

The electromotive force $V_{e m f}$ that induces the armature is proportional to the angular velocity. 


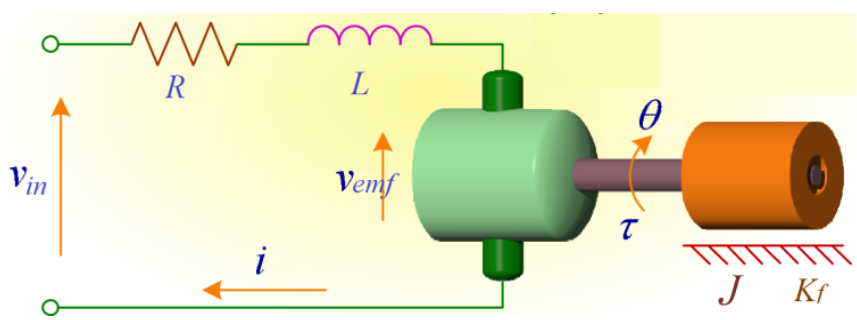

Fig. 1 DC motor representation circuit.

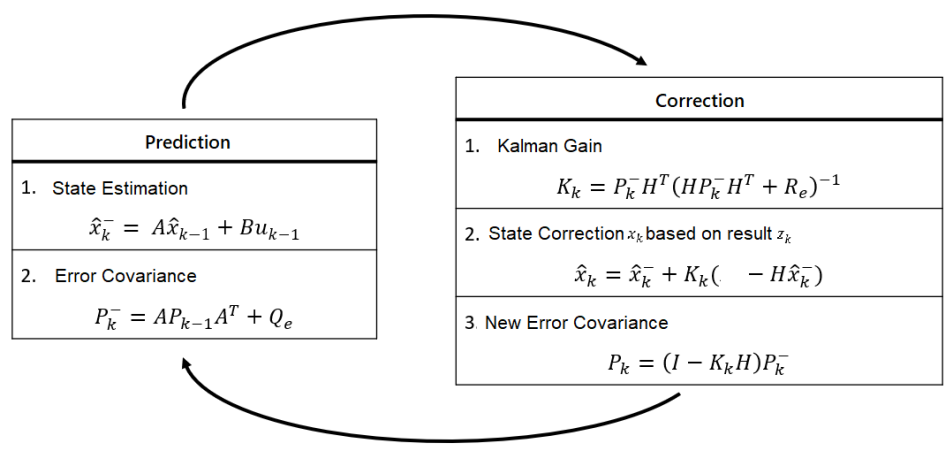

Fig. 2 Kalman filter algorithm.

$$
V_{e m f}(t)=K_{b} \omega(t)
$$

where $K_{b}$ denotes a constant of the electromotive force which value is determined by the DC motor's physical properties.

Newton's law lowers the torque acting on the DC motor. The sum of all torque in the motor equals the inertia load times $(J)$ the derivative of the angular speed, as in (3).

$$
\Sigma \tau_{i}=J \frac{d \omega(t)}{d t}=-K_{f} \omega(t)+K_{m} i(t)
$$

where $K_{f} \omega(t)$ denotes the friction force.

The equation for the armature is derived by the voltage Kirchoff law as in (4).

$$
V_{\text {in }}(t)-V_{\text {emf }}(t)=L \frac{d i(t)}{d t}+R i(t)
$$

By substituting (2) to (4), it is obtained (5).

$$
V_{\text {in }}(t)=L \frac{d i(t)}{d t}+R i(t)+K_{b} \omega(t)
$$

Two differential equations are obtained from the description above that describes a DC motor's dynamic properties in Fig. 1, namely (6) and (7). Equation (6) is the relationship between the change in the induced current to the input voltage $\left(V_{i n}\right)$.

$$
\frac{d i(t)}{d t}=-\frac{R}{L} i(t)-\frac{K_{b}}{L} \omega(t)+V_{i n}(t) .
$$

Meanwhile, (7) describes the relationship between the motor rotation acceleration to the induced current $(i)$ as can be seen in (7).

$$
\frac{d \omega(t)}{d t}=\frac{K_{m}}{J} i(t)-\frac{K_{f}}{L} \omega(t) .
$$

The relationship between angular velocity $(\omega)$ and input voltage $\left(V_{\text {in }}\right)$ can be obtained by (6) and (7).

In designing the control system, the plant model to be controlled is made in a transfer function or state space. By selecting current $i(t)$ and angular speed $\omega(t)$ as state variables, input voltage $\left(V_{i n}\right)$ as system input and angular velocity $\omega(t)$ as system output, a DC motor state-space representation is obtained as follows.

$$
\begin{gathered}
\frac{d}{d t}\left[\begin{array}{c}
i(t) \\
\omega(t)
\end{array}\right]=\left[\begin{array}{cc}
-\frac{R}{L} & -\frac{K}{L} \\
\frac{K_{m}}{J} & -\frac{K_{f}}{J}
\end{array}\right]\left[\begin{array}{c}
i(t) \\
\omega(t)
\end{array}\right]+\left[\begin{array}{c}
\frac{1}{L} \\
0
\end{array}\right] V_{i n}(t) . \\
y(t)=\left[\begin{array}{ll}
0 & 1
\end{array}\right]\left[\begin{array}{c}
i(t) \\
\omega(t)
\end{array}\right]+[0] V_{i n}
\end{gathered}
$$

\section{PID CONTROL}

A PID control is a combined control system between proportional (P), integral (I), and derivative (D) control [9]. The PID control system in the time domain is stated as follows.

$$
\begin{aligned}
C(t) & =P+I+D \\
& =K_{p} e(t)+K_{i} \int_{0}^{t} e(t) d t+K_{d} \frac{d e(t)}{d t} .
\end{aligned}
$$

$C(t)$ indicates the control output, $K p$ indicates a proportional constant, $K i$ indicates an integral constant, $K d$ indicates a derivative constant, and $e$ indicates a system error which is the input for the PID.

Proportional control (P) functions to reduce the rise time or rise time, however it does not eliminate the steady-state error. Integral control (I) has a function of eliminating steady-state errors without affecting the transition response. On the other hand, derivative control (D) has functions including increasing system stability, reducing overshoot, but decreasing transition response.

\section{KALMAN FILTER}

Kalman filter has been widely used in various applications such as robotics, navigation, and vehicle control systems [10]. 


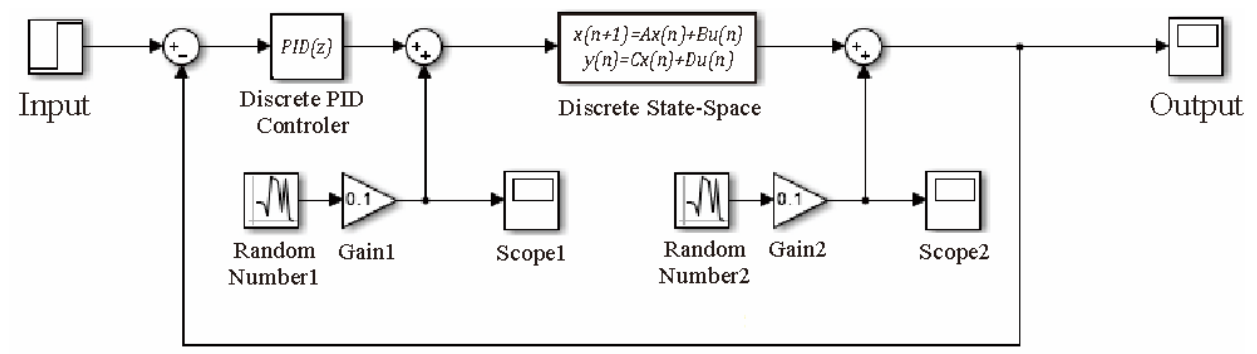

Fig. 3 System block diagram with PID.

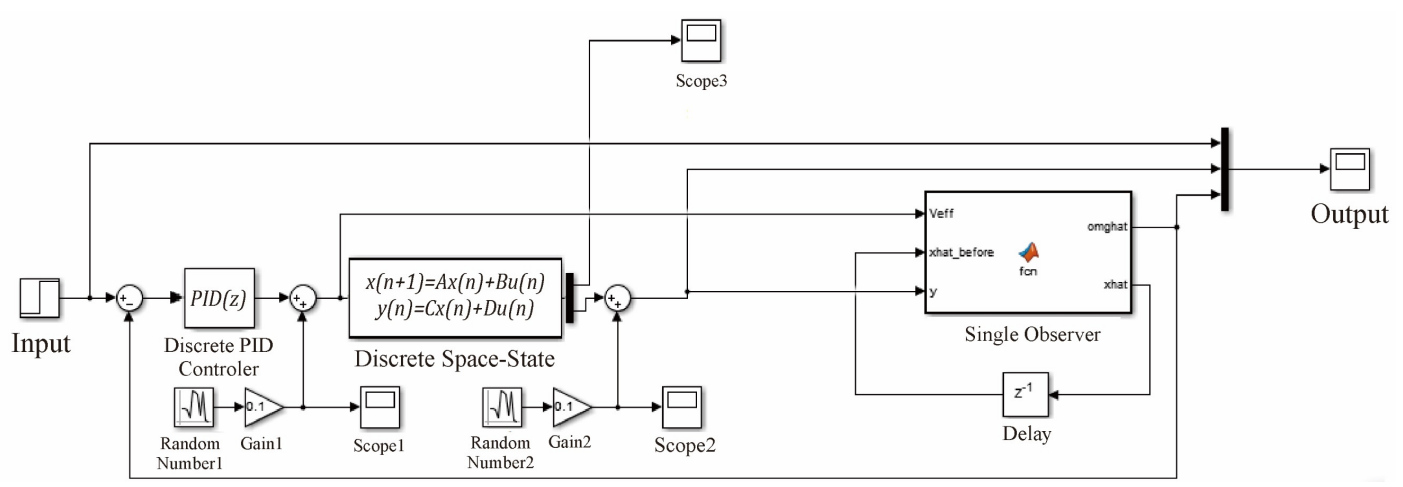

Fig. 4 Diagram of system block with PID + observer.

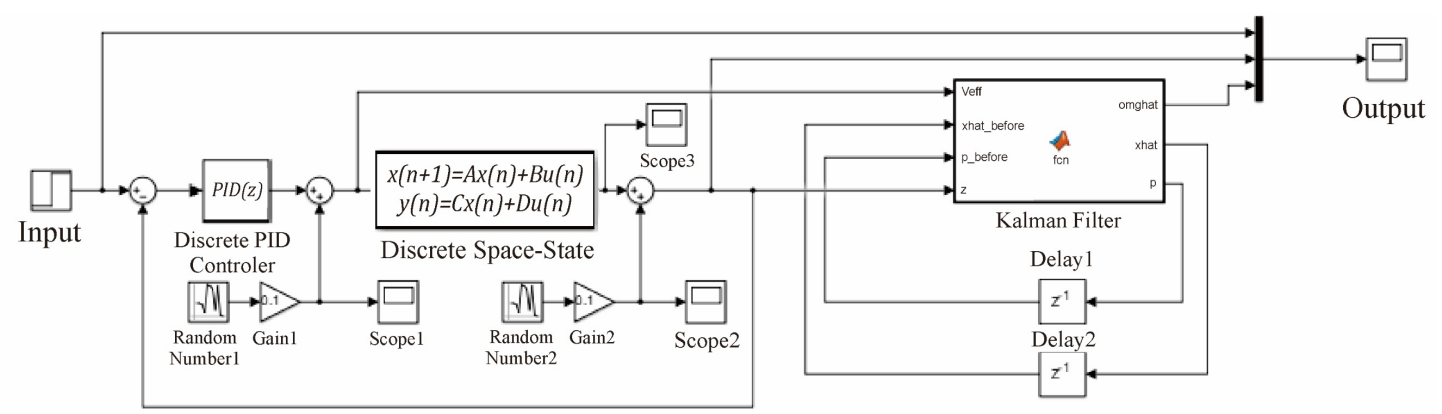

Fig. 5 Diagram of system block with PID + Kalman filter.

Kalman filter itself is one type of estimator that is reliable in handling noise [11]. It uses a series of continuous measurement data which has noise and other measurement inaccuracies. It produces variable estimates which values tend to be more precise than the measurement results.

The design of the Kalman filter depends on the plant model used. In this study, the DC motor model is represented in statespace (8) and (9). In general form, discrete state space can be written as in (11).

$$
x_{k}=A x_{k-1}+B u_{k}+w_{k} .
$$

$A$ is the transition matrix used to derive the current state variable $\left(x_{k}\right)$ based on the previous state variable $\left(x_{k-1}\right)$. Then, $B$ and $u_{k}$ are the input vector and system input, respectively. At the same time, $w_{k}$ is process noise, with covariance $R_{e}$.

Meanwhile, the measurement results are modeled with variables $z_{k}$. It can be seen in (12).

$$
z_{k}=H x_{k}+v_{k}
$$

$H$ is the output vector and $v_{k}$ is the measurement noise, with covariance $Q_{e}$ [12]. Each noise is good $w_{k}$. Moreover, $v_{k}$ is assumed to be independent and have probability of a normal distribution with a mean of 0 or called Additive white Gaussian noise.

The Kalman filter algorithm consists of two processes, namely prediction and correction. The recursive algorithm of the discrete Kalman filter can be seen in Fig. 2. Decreasing the formula used in Kalman filter can be seen in [12].

\section{Simulation}

The simulation design compares the usual PID control with Kalman PID + filter control. Fig. 3 is a block diagram of the control system using only PID, Fig. 4 is a is a control system that uses a PID + observer, while Fig. 5 is a control system that uses a PID + Kalman filter. The simulation was designed by adding noise to the control system and its output. Undesirable noise in the entire system would affect the output and cause the system not to operate optimally.

In this simulation, process noise and measurement noise were assumed to be independent of each other and had a normal probability distribution with a mean of 0 . The control system's performance was tested through a step response so that the 


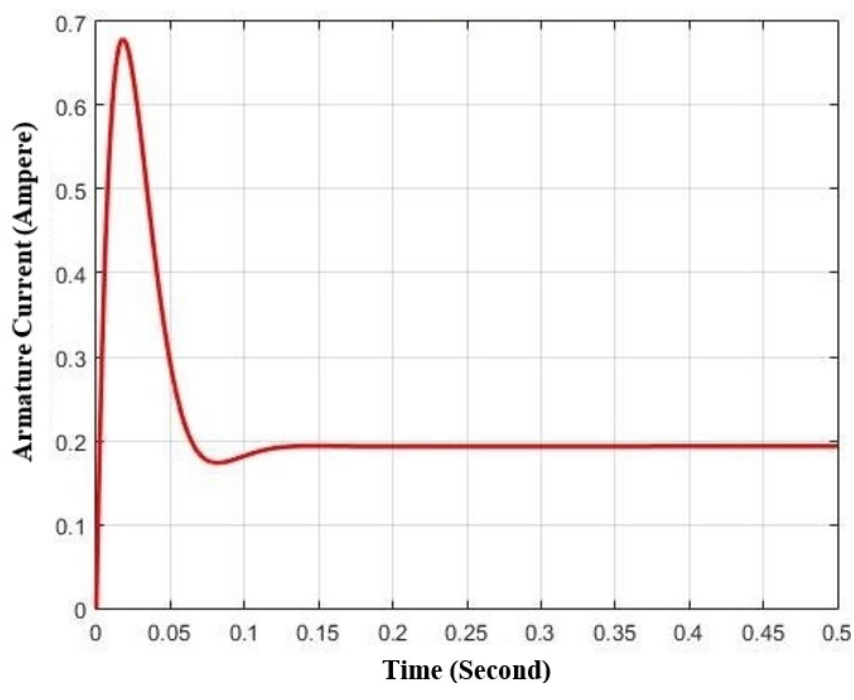

Fig. 6 Ideal armature current flow chart.

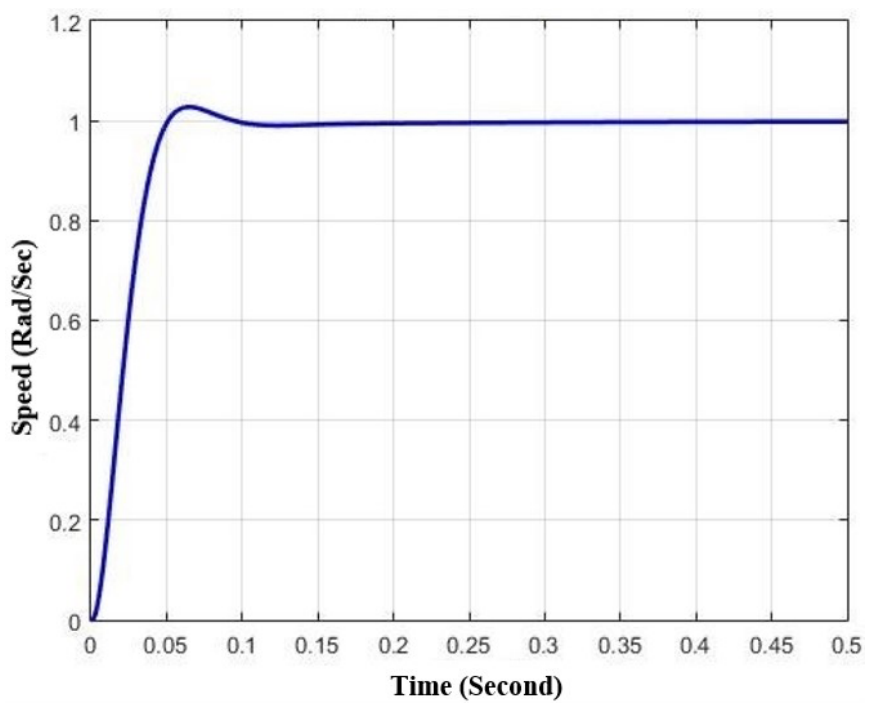

Fig. 7 Graph of ideal motor speed without noise.

reference signal was set at $1 \mathrm{rad} / \mathrm{sec}$. The parameters $K p, K i$, and $K d$ were set at 5,2, and 0.08 , respectively.

\section{RESUlT AND DisCUSSION}

The simulation was divided into four scenarios. These scenarios are the DC motor control system in ideal conditions, the PID control system that assumed there was process noise and measurement noise, the Kalman PID + observer control system, and the Kalman PID + filter control system that assumed there was process noise and measurement noise. The second until four scenarios were made to test the PID control system's performance on a DC motor in real conditions since the noise will always interfere with the system in the field.

As previously mentioned, a DC motor has two states, namely armature current and speed. In the first scenario, the DC motor's armature current and speed response is shown in Fig. 6 and Fig. 7. At the start of the time, the armature current rises sharply then slope to its nominal value of 0.2 ampere. Based on (3), the amount of torque is affected by the current. The increase

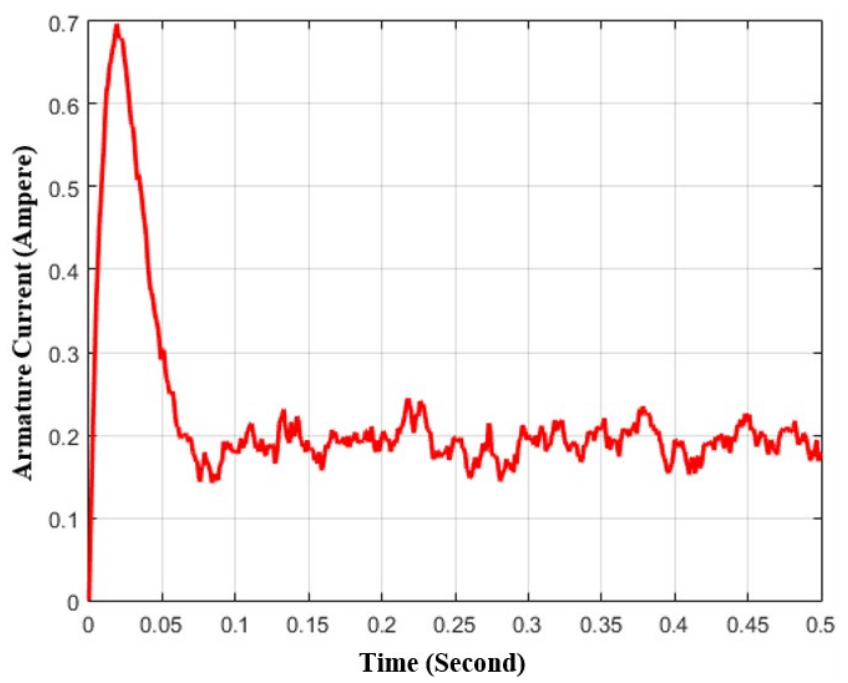

Fig. 8 Graph of armature current when there is noise.

in current at the beginning of the simulation time occurred because the motor requires a large enough torque at the simulation's beginning.

Ideal speed means that processing noise or measurement noise does not occur. The ideal speed in Fig. 7 shows that the PID control system can work appropriately, with a settling time of 0.1 second and an overshoot of $2.9 \%$. Moreover, the steadystate error also does not appear in this scenario.

In the second scenario, there were the addition of measurement noise and process noise to the system. Due to noise, sensor readings were inaccurate. In the second scenario, the current entering the armature had a lot of ripples with a high enough frequency, as shown in Fig. 8. When implemented in a real plant, such control system will accelerate plant damage due to current ripples.

The PID control has an input in the form of an error, which is the difference between the sensor reading and the reference signal. If the sensor readings are hit by noise, the PID input will not be accurate. Moreover, it will generate an incorrect control signal and tend to ripple.

The ripple control signal plus the presence of process noise causes the motor speed to ripple, as shown in Fig. 9. If it is not handled properly, such control system will lead to instability. This motor speed response is eminently avoided in the industrial world since it is inefficient and accelerates component breakdown. DC motor power is channeled using a conveyor belt or gear set. The speed response, which has a lot of overshoots, will cause the gear teeth to wear out quickly. In addition to that, the electrical energy needs to operate a DC motor more, so it will be inefficient. It needs a control system which has characteristics such as resistant to noise or capable of filtering noise. Therefore, the arisen effects of the system can be minimized.

Based on Fig. 9, the motor speed continues to oscillate with a reasonably high frequency. This response is not a natural response from a DC motor but the effect of noise. The sensor that reads the motor speed is boarded by the reading noise so that the motor speed continues to oscillate and does not go to 


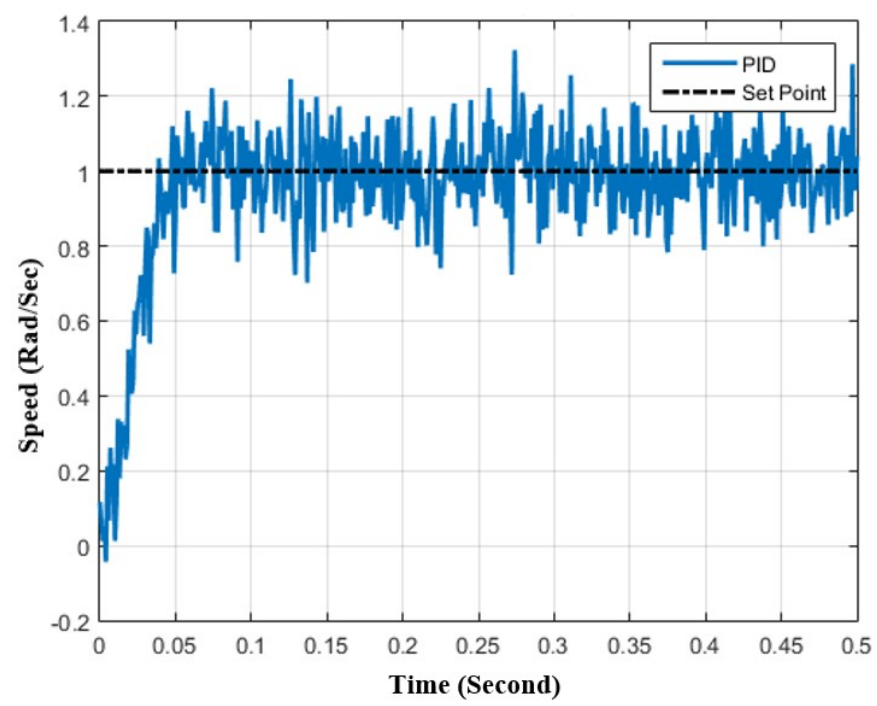

Fig. 9 Graph of motor speed with noise on PID controller.

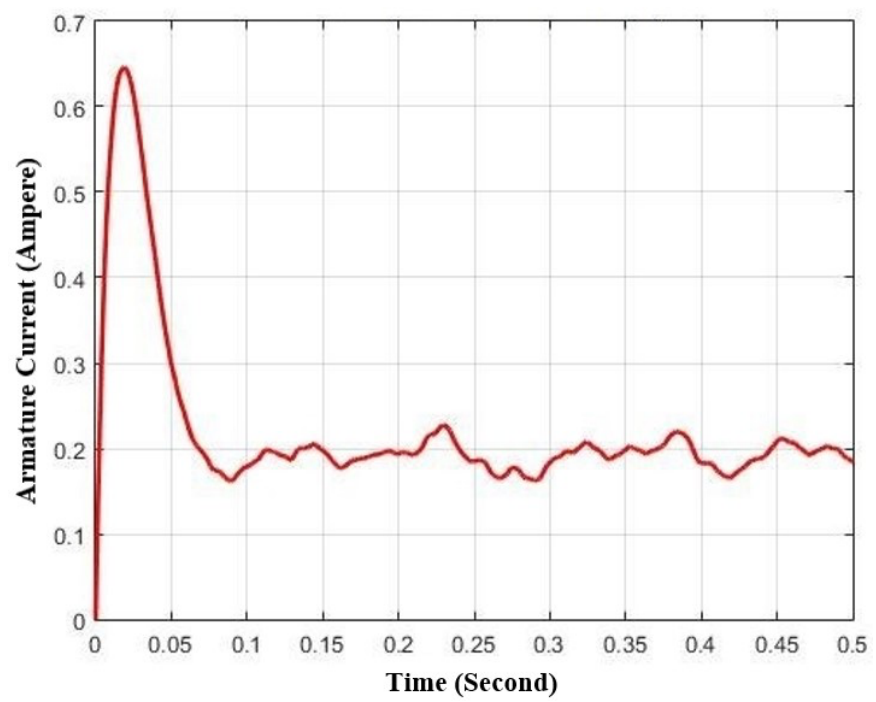

Fig. 10 Armature flow chart when observer is implemented.

the set point. Thus, this scenario's DC motor speed will continue to have a steady-state error, and the settling time will never be reached.

On the third scenario, an observer was added to system. The observer acted as an estimator to predict the amount of system when there were no noises. On this third scenario, the feedback was not taken from the sensor, but from the result of process from observer, as shown in Fig. 4. The output from the armature and speed can be seen on Fig. 10 and Fig. 11.

The armature flows on the third scenario is showed by Fig. 10. Same as before, the flux on this scenario had ripples but it became more limited if it was compared to the second scenario with PID controller. It occurred as the state estimation that was conducted by observer made the value more accurate if compared only with the results of the reading sensor.

The speed on the third scenario showed by Fig. 11. Same as before, the flux on this scenario had ripples but it became more limited if it was compared to the second scenario with PID controller. It occurred since the state estimation that conducted

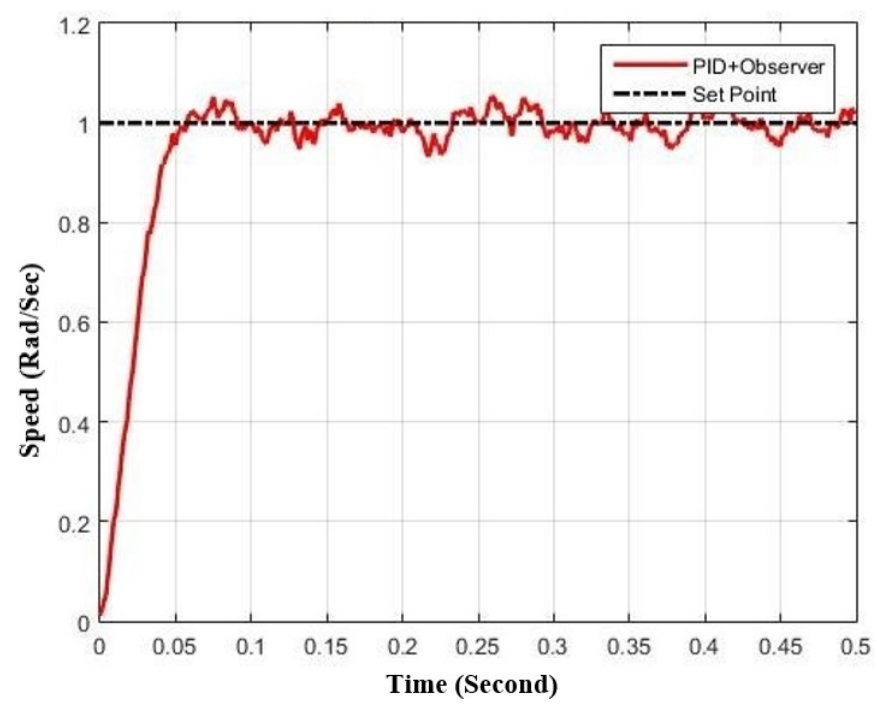

Fig. 11 Motor speed graph with noise on PID controller + observer.

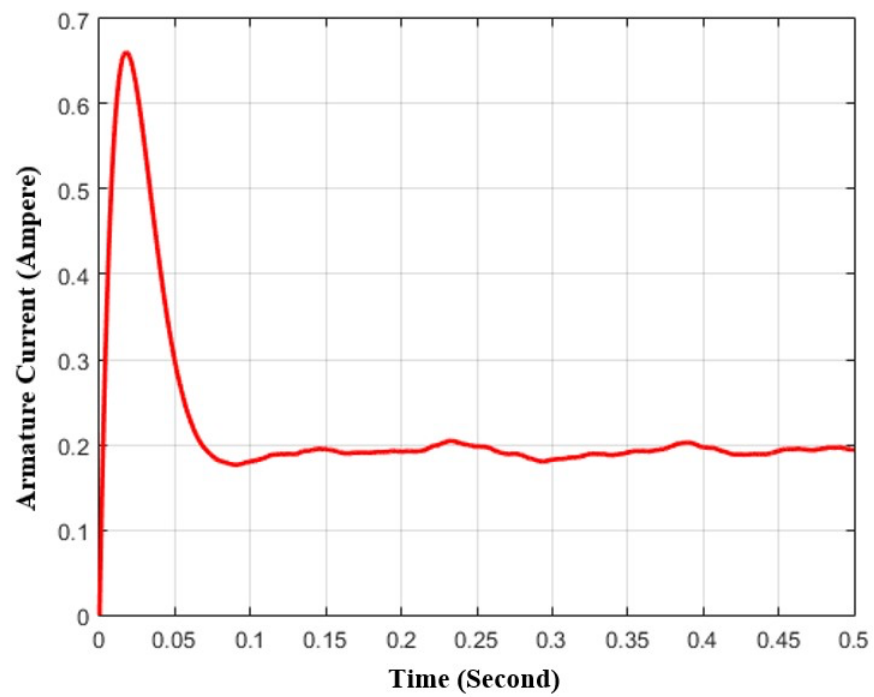

Fig. 12 Armature flow chart when Kalman filter is implemented.

by observer made the value more accurate if compared only with the results of reading sensor.

In the fourth scenario, Kalman filter was added to the system. It functioned as an estimator to predict the amount of system output when there was no noise. In the third scenario, the feedback was not taken from the sensor, but from Kalman filter estimation results, as shown in Fig. 5.

The armature current in scenario four is shown in Fig. 12. Unlike the second scenario, the currents in this scenario did not have ripples since the state estimation carried out by the Kalman filter produced a more accurate value when compared to the sensor readings alone.

A better state estimation will produce a better control signal, which is reflected in the motor speed response, which tends not to ripple, as shown in Fig. 13. This speed response is similar to the speed response of a DC motor under ideal conditions. The resulting overshoot was less than $2 \%$, with a settling time of 0.1 second without any steady-state error. From these results, it can be seen that the PID control system designed can work well 


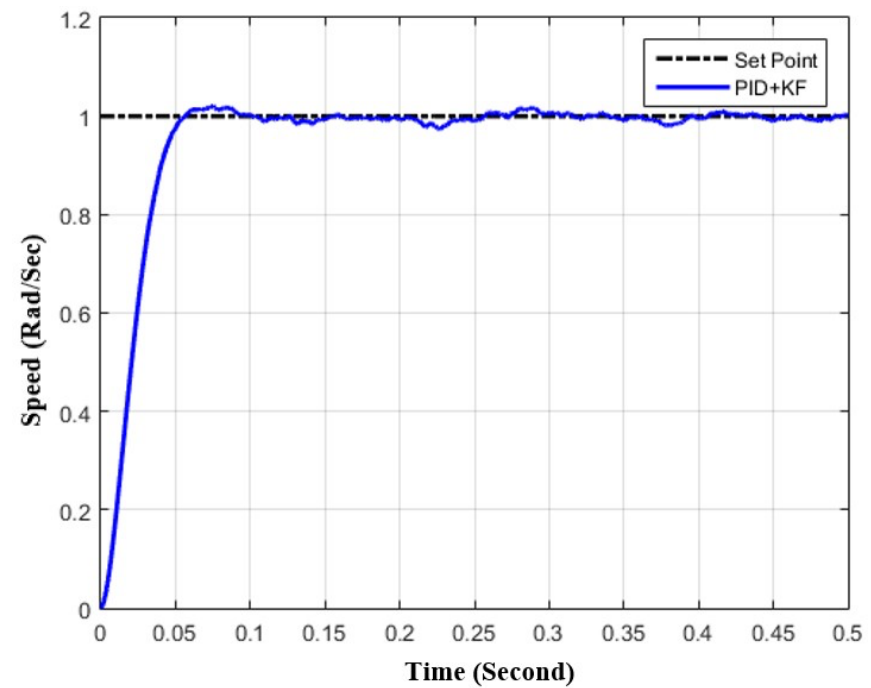

Fig. 13 Motor speed graph with noise on PID controller + Kalman filter.

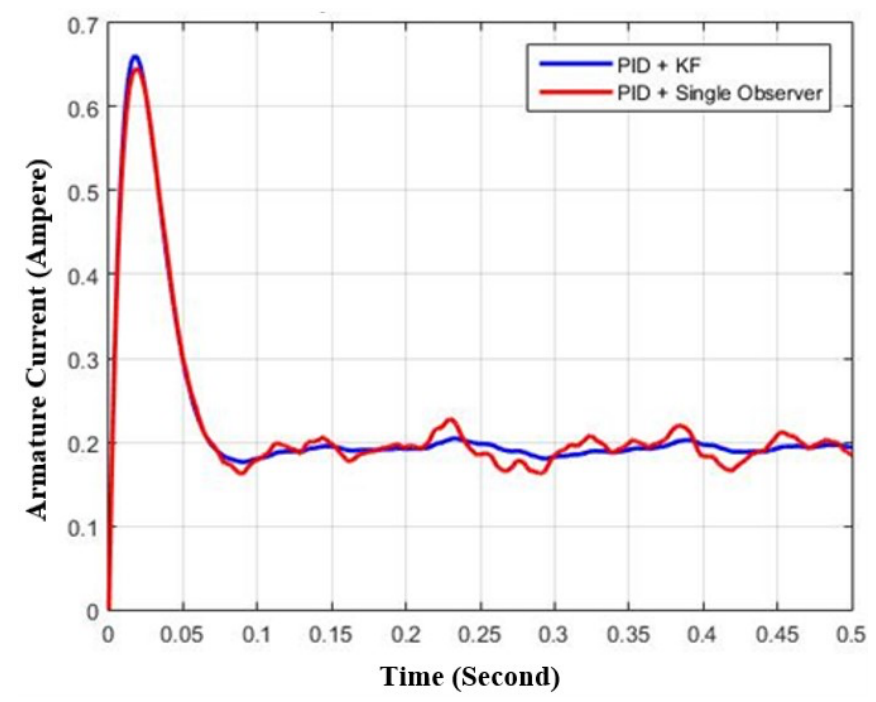

Fig. 14 Comparison graph of motor Armature current with PID + observer and $\mathrm{PID}+\mathrm{KF}$.

even though there is process noise and measurement noise. Comparison between flows and speed with PID + KF controller and PID + observer controller can be seen on Fig. 14 and Fig. 15. From Fig. 14, the comparison output from armature flows between Tapis Kalman and observer shows significant differences. It seemed that PID controller with Tapis Kalman showed a better result in minimizing noises on control system of DC motor.

Based on Fig. 15, the comparison output from DC motor speed between Tapis Kalman and observer shows significant differences. It seems that PID controller with Tapis Kalman shows better result in minimizing noises on DC motor control system.

Furthermore, it is necessary to test the Kalman filter's effectiveness in the PID control system. The test was conducted by comparing the motor speed in second scenario with the motor speed in third scenario and motor speed in fourth scenario. The parameters used to test were the transient

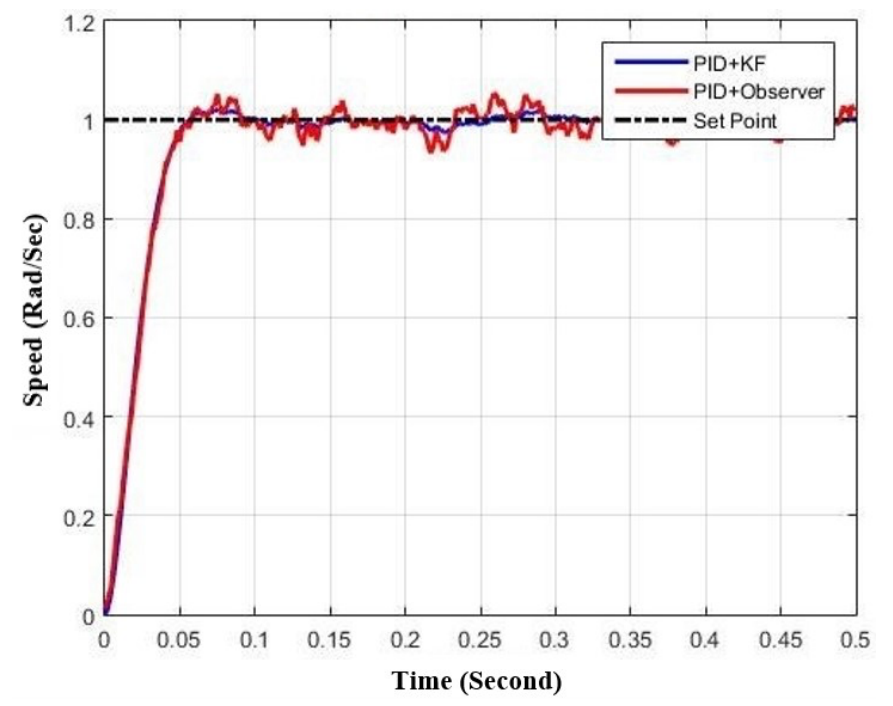

Fig. 15 Comparison graph of motor speed with PID + observer and PID + KF.

TABLE I

COMPARISON OF CONTROL SYSTEM PERFORMANCE

\begin{tabular}{|c|c|c|c|}
\hline \multirow{2}{*}{ Type of Control } & \multicolumn{3}{|c|}{ Test Parameter } \\
\cline { 2 - 4 } & Settling Time & Overshoot & IAE \\
\hline PID & $>0.5$ second & $27.2 \%$ & 60.9 \\
\hline PID + observer & $>0.5$ second & $2.1 \%$ & 31.0 \\
\hline PID + KF & 0.1 second & $1.8 \%$ & 25.5 \\
\hline
\end{tabular}

response (settling time and overshoot) and IAE. The comparison of the speed response in the third and fourth scenarios can be seen in Fig. 15. At the same time, the test results can be seen in Table I.

\section{CONCLUSION}

The method of improving PID performance with Kalman filters on DC motors has been successfully developed. From the research results, in the form of simulations that have been made, it is concluded that the performance of PID with filters has a better performance than ordinary PID in minimizing the existing noise. Kalman filter can estimate the DC motor state correctly so that the resulting response also resembles an ideal DC motor. By implementing the Kalman filter in the PID control system, the motor speed response is better than the PID control without the Kalman filter. The test results showed that the PID control system had an IAE of 60.9. PID controller with the observer had an IAE of 31.0. Simultaneously, the combination of PID and Kalman filter was superior to the IAE with 25.5.

\section{REFERENCES}

[1] K. Sharma and D.K. Palwalia, "A Modified PID Control with Adaptive Fuzzy Controller Applied to DC Motor," 2017 International Conference on Information, Comunication, Instrimentation and Control, 2017, pp. 16.

[2] K. Singh, P. Vasant, I. Elamvazuthi, and R. Kannan, "PID Tuning of Servo Motor Using Bat Algorithm," Procedia Computer Science, Vol. 60, No. 1, pp. 1798-1808, Sep. 2015.

[3] P.M. Meshram and R.G. Kanojiya, "Tuning of PID Controller Using Ziegler-Nichols Method for Speed Control of DC Motor," International 
Conference on Advances in Engineering, Science and Management, 2012, pp. 117-122.

[4] E.A. Ebrahim, "Ant-Colony Optimization Control of Brushless-DC Motor Driving a Hybrid Electric-Bike and Fed from Photovoltaic Generator," 2016 IEEE Congress on Evolutionary Computation, 2016, pp. 4221-4228.

[5] A. Jaya, E. Purwanto, M.D. Fauziah, F.D. Murdianto, G. Prabowo, and M.R. Rusli, "Design of PID-Fuzzy for Speed Control of Brushless DC Motor in Dynamic Electric Vehicle to Improve Steady-State Performance," 2017 International Electronics Symposium on Engineering Technology and Applications, 2017, pp. 179-184.

[6] [S. Abedini and H. Zarabadipour, "Tuning of an Optimal PID Controller with Iterative Feedback Tuning Method for DC Motor," The 2nd International Conference on Control, Instrumentation and Automation, 2011, pp. 611-615.

[7] [Z. Has, A.H. Muslim, and N.A. Mardiyah, "Adaptive-Fuzzy-PID Controller Based Disturbance Observer for DC Motor Speed Control," 2017 4th International Conference on Electrical Engineering, Computer Science and Informatics, 2017, pp. 1-6.
[8] C. Urrea and J. Kern, "A New Model for Analog Servo Motors. Simulations and Experimental Results," Canadian Journal on Automation, Control and Intelligent Systems, Vol. 2, No. 2, pp. 29-38, Mar. 2011.

[9] R. Akbari-Hasanjani, S. Javadi, and R. Sabbaghi-Nadooshan, "DC Motor Speed Control by Self-Tuning Fuzzy PID Algorithm," Transactions of the Institute of Measurement and Control, Vol. 37, No. 2, pp. 164-176, Feb. 2015.

[10] S. Praesomboon, S. Athaphaisal, S. Yimman, R. Boontawan, and K. Dejhan, "Sensorless Speed Control of DC Servo Motor Using Kalman Filter," 2009 7th International Conference on Information, Communications and Signal Processing, 2009, pp. 1-5.

[11] M. Marshall and H. Lipkin, "Kalman Filter Visual Servoing Control Law," 2014 IEEE International Conference on Mechatronics and Automation, 2014, pp. 527-534.

[12] G. Welch and G. Bishop, “An Introduction to the Kalman Filter," Univ. of North Carolina at Chapel Hill, Chapel Hill, NC, TR 95-041, pp. 1-16, 2006. 